\title{
Uni-directional waves over slowly varying bottom. Part I: Derivation of a KdV-type of equation
}

\author{
E. van Groesen \\ University of Twente, Department of Applied Mathematics, Enschede, The Netherlands
}

S.R. Pudjaprasetya ${ }^{1}$

Institut Teknologi Bandung, Department of Mathematics, Bandung, Indonesia

Received 5 August 1992, Revised 14 May 1993

\begin{abstract}
The exact equations for surface waves over an uneven bottom can be formulated as a Hamiltonian system, with the total energy of the fluid as Hamiltonian. If the bottom variations are over a length scale $L$ that is longer than the characteristic wavelength $\ell$, approximating the kinetic energy for the case of "rather long and rather low" waves gives Boussinesq type of equations. If in the case of an even bottom one restricts further to uni-directional waves, the Korteweg-de Vries (KdV) is obtained. For slowly varying bottom this uni-directionalization will be studied in detail in this part $I$, in a very direct way which is simpler than other derivations found in the literature. The surface elevation is shown to be described by a forced KdV-type of equation. The modification of the obtained KdV-equation shares the property of the standard KdV-equation that it has a Hamiltonian structure, but now the structure map depends explicitly on the spatial variable through the bottom topography. The forcing is derived explicitly, and the order of the forcing, compared to the first order contributions of dispersion and nonlinearity in $\mathrm{KdV}$, is shown to depend on the ratio between $\ell$ and $L$; for very mild bottom variations, the forcing is negligible. For localized topography the effect of this forcing is investigated. In part II the distortion of solitary waves will be studied.
\end{abstract}

\section{Introduction}

Consider a layer of incompressible fluid over an uneven bottom. Assuming the flow to be irrotational, the motion of surface waves under the influence of gravity is given by a (generalised) Hamiltonian system in the variables $\eta(x, t)$ and $u(x, t)$, where $\eta(x, t)$ is the wave height measured from an undisturbed water level and $u(x, t)$ is the spatial derivative of the restriction to the surface of the velocity potential. The Hamiltonian is the total energy of the fluid. Considering waves above an even bottom with wavelength $\ell$ and amplitude $a$, which satisfy $a=\mathrm{O}(\epsilon)$ and $1 / \ell^{2}=\mathrm{O}(\epsilon)$, an approximation of the kinetic energy leads to Boussinesq type equations, which describe waves running in both directions. For even bottom, one can restrict Boussinesq equations to an equation for uni-directional waves along the same line as outlined below for the uneven bottom. Then one gets as characteristic approximation the well-known $\mathrm{KdV}$ equation. This equation is of the form

$$
\partial_{t} \eta=-\Gamma_{0} \delta_{\eta} H_{0}(\eta)+\mathrm{O}\left(\epsilon^{2}\right), \text { with } \Gamma_{0}=c_{0} \partial_{x},
$$

1 Now at University of Twente, Department of Applied Mathematics, Enschede, The Netherlands.

0165-2125/93/\$06.00 (C) 1993 - Elsevier Science Publishers B.V. All rights reserved 
where $c_{0}$ is related to the constant depth $h_{0}$ by $c_{0}=\sqrt{g h_{0}}$ with $g$ the acceleration of gravity. The Hamiltonian $H_{0}(\eta)$ is a functional of $\eta$ and is conserved during the evolution (constant of the motion), if in (1) the $O\left(\epsilon^{2}\right)$ terms are left out. Explicitly it is given by

$$
H_{0}(\eta)=\int \frac{1}{2} \eta^{2}+\epsilon k_{0}(\eta)
$$

where $k_{0}(\eta)$ are the first order contributions due to dispersion and nonlinearity, measured by the small quantity $\epsilon$.

For uneven bottom, let $h(x)$ be the depth of the fluid describing the bottom topography and let

$$
c(x)=\sqrt{g h(x)} \text {. }
$$

In this paper, we will consider slow bottom variations satisfying

$$
\frac{h^{\prime}(x)}{h(x)}=\mathrm{O}\left(L^{-1}\right), \text { and } h^{\prime}(x)=\mathrm{O}\left(L^{-1}\right), \text { with } L=\ell^{1+2 \alpha} .
$$

Here, $L$ is a characteristic length scale of the bottom variations. Whenever $\alpha>0$ it is found that a direct generalization of Boussinesq equation is valid: the constant depth just being replaced by the varying quantity. Note that $\alpha>0$ means that the bottom variations are over a longer length scale than the characteristic wavelength $\ell$.

In the procedure to find an equation for the surface elevation for uni-directional waves, we write the elevation $\eta$ as

$$
\eta=r-s
$$

and a corresponding linear combination for $u$. Then Boussinesq equations can be reformulated as a coupled set of equations for $r$ and $s$ of the form:

$$
\partial_{t} r=-\Gamma(x) \delta_{r} H(r, s)-\frac{1}{2} c^{\prime} \delta_{s} H, \quad \text { with } \quad \Gamma(x)=\frac{1}{2}\left(c(x) \partial_{x}+\partial_{x} c(x)\right)
$$

and a corresponding one for $s$. In lowest order, $r$ describes waves running to the right, while $s$ accounts for waves running to the left. This set of coupled equations has a Hamiltonian structure just like the equivalent Boussinesq equations. And, just like $\Gamma_{0}$, the operator $\Gamma(x)$ that appears, is a skew-symmetric operator, now depending explicitly on $x$.

When we consider initial data for which the contribution of left running waves is small and when $c^{\prime}$ is very small, $s$ can be taken equal to zero in (5), leading to

$$
\partial_{t} r=-\Gamma(x) \delta_{r} \bar{H}(r),
$$

with $\bar{H}(r)=H(r, s=0)$. This equation for $r$ is a modification of the standard $\mathrm{KdV}$ equation: it reduces to the standard $\mathrm{KdV}$ equation in the case of an even bottom. Moreover, it has a Hamiltonian structure due to the fact that $\Gamma(x)$ is skew-symmetric. As a consequence, the Hamiltonian $\bar{H}(r)$ is conserved. The Hamiltonian reads like (2)

$$
\bar{H}(r)=\int \frac{1}{2} r^{2}+\epsilon k(r)
$$

but now $k(r)$ depends on the topography. The linear equation that appears when in (6) the effects of dispersion and nonlinearity are neglected in the Hamiltonian (putting $\epsilon=0$ in (7)) can be solved 
analytically. The solutions show implicitly the main features of the structure map $\Gamma$ : an increase in amplitude and a decrease of wavelength and velocity for waves running into shallower water, in agreement with common expectations. (In part II the distortion of solitary waves will be described.)

It turns out that the surface elevation given by the linear combination (4) contains a contribution of $s$ that cannot be neglected in general. However, for initial data that represent uni-directional waves, the $s$-contribution satisfies a linear equation in the lowest order and can be solved explicitly. Then, with $r$ satisfying the $\mathrm{KdV}$-type of eq. (6), the surface elevation can be found. It will be shown that $\eta$ satisfies the same equation (6) with a specific forcing:

$$
\partial_{t} \eta=-\Gamma(x) \delta_{\eta} \bar{H}(\eta)-\mathcal{F}+\mathrm{O}\left(\epsilon^{1+\alpha}\right) .
$$

The forcing $\mathcal{F}$ is derived explicitly and can be calculated from the initial elevation. The order of the forcing depends strongly on the ratio between wavelength $\ell$ and the length $L$ over which bottom variations take place. For $\alpha \geqslant 1$ (so, $L \geqslant \mathrm{O}\left(\ell^{3}\right)$, very mild bottom variation), the forcing $\mathcal{F}$ is in negligible order $\mathrm{O}\left(\epsilon^{2}\right)$. This equation has a Hamiltonian structure; conserved integrals are energy and mass (not momentum!). In the limiting case of an even bottom it reduces to the familiar $\mathrm{KdV}$ equation. For $0<\alpha<1$, the forcing is in significant order $\mathrm{O}\left(\epsilon^{2 \alpha}\right)$. If $\frac{1}{2}<\alpha<1$, the Boussinesq terms dominate the forcing $\mathcal{F}$, and we obtain the $\mathrm{KdV}$-type of equation with higher order forcing. If $0<\alpha<\frac{1}{2}$, the forcing $\mathcal{F}$ dominates the Boussinesq effect, and we have a linear equation which is correct up to $\mathrm{O}(\epsilon)$. An approximate solution for the linear equation can be obtained. In the special case of localized bottom variations, we show that this forcing is localized too. For an initial wave upstream, this force may be active for a finite or infinite time, depending on the value of a certain mass-like quantity.

The organization of the paper is as follows. In Section 2 we briefly recall the Hamiltonian formulation of the exact equations for surface waves. This formulation for waves on a layer of infinite depth was given by Zakharov [1], and for finite depth by Broer [2] (see also Broer et al. [3]). In Section 3 the approximation leading to the bi-directional Boussinesq equation is described. For constant depth the derivation based on retaining the Hamiltonian structure was already given in [2] and [3]. In Section 4, firstly we resume from [3] the derivation of the KdV-equation for the case of constant bottom, in a way that is most suited for the generalization to uneven bottom. A somewhat different method to derive KdV from Boussinesq for even bottom can be found in Olver [4]. See also Mattioli [5] who retains the coupling between the KdV-equation for right and left travelling waves. Olver [6] discusses various approximations with or without Hamiltonian structure. Then, the linear lowest order equation is investigated, which also shows the special properties of the map $\Gamma(x)$. Finally, we derive the $\mathrm{KdV}$-type of the equation for slowly varying bottom which generalizes the usual $\mathrm{KdV}$. In Section 5 we derive the forced $\mathrm{KdV}$ equation (8) for the elevation itself and investigate the forcing in case of localized bottom variations.

The way in which eq. ( 8 ) is obtained in this paper is very direct: the forcing $\mathcal{F}$ is expressed explicitly in contributions from left-travelling waves due to unevenness. This is different from many derivations in literature, where equations are usually derived from a series substitution for the potential, leading to a forced KdV-type of equation for the primary coefficient in the series expansion. The assumptions underlying the various approximations are less clearly separated from the truncation argument. Moreover, the transformations involved do not help to clarify the essential underlying model. Newell [8] and Grimshaw [9] derived equations for quantities related to, but different from the actual surface elevation, including expressions for the forcing. For clarity, we resume in Section 6 Newell's result, 
which deals with a particular case of topography $(\alpha=1)$. Comparison with our result shows a complete agreement. Mei in Ref. [7] resumes several perturbation methods. The equation derived there is based on the previous work of Madsen and Mei [10] and is related to an equation obtained by Kakutani [11] and Johnson [12]. Miles [13] made some observations about conserved properties for the energy and momentum of those equations. For the evolution of a wave train, Djordjević and Redekopp [14] derived a Schrödinger like equation for the envelope. Although in special cases these equations may be correct to the same order of approximation, they lack the property of the equation to be derived here which retains the Hamiltonian structure. And as stated before, the forcing derived here allows a precise estimate for its order of magnitude in comparison to the effect of dispersion and non-linearity in the KdV equation. And finally, we have the conclusions in Section 7.

\section{Hamiltonian formulation}

Consider a layer of incompressible fluid over an (un)even bottom. Let the function $h(x)$ describe the bottom topography, and $\eta(x, t)$ the surface elevation measured from the undisturbed level $z=0$, see Fig. 1. Let $\Omega(\eta)$ denote the domain of the fluid i.e.

$$
\Omega(\eta)=\left\{(x, z) \in R^{2} \mid-h(x) \leqslant z \leqslant \eta(x, t)\right\} .
$$

Assuming that the flow is irrotational, the particle velocity can be written like $v(x, z)=\nabla \Phi(x, z)$, with $\Phi(x, z)$ the fluid potential. The fluid is incompressible, so $\div v=0$ in $\Omega(\eta)$ or

$$
\triangle \Phi=0, \quad \text { in } \Omega(\eta)
$$

At the bottom, the normal component of the velocity is zero, hence $\boldsymbol{v} \cdot \boldsymbol{n}=0$ with $\boldsymbol{n}$ the normal vector, or

$$
\nabla \Phi \cdot \boldsymbol{n}=0, \text { for } z=-h(x)
$$

The momentum equation (Euler equation) leads to the Bernoulli equation, which restricted to the free surface (assuming the pressure above the layer is atmospheric) reads:

$$
\partial_{t} \Phi=-\frac{1}{2}|\nabla \Phi|^{2}-g \eta, \quad \text { at } \quad z=\eta(x, t),
$$

with $g$ the gravitational acceleration. Finally, the condition that no particles flow through the surface leads to the kinematic boundary condition:

$$
\partial_{l} \eta=\nabla \boldsymbol{\Phi} \cdot \boldsymbol{n}, \quad \text { at } \quad z=\eta(x, t) .
$$

The four equations $(9)-(12)$ are the equations describing the motion of the surface waves.

At the surface $z=\eta(x, t)$, the value of the potential is denoted by $\varphi(x, t) \equiv \Phi(x, \eta(x, t), t)$. The Hamiltonian $H(\varphi, \eta)$ is the total energy functional,

$$
H(\varphi, \eta)=K(\varphi, \eta)+P(\eta),
$$




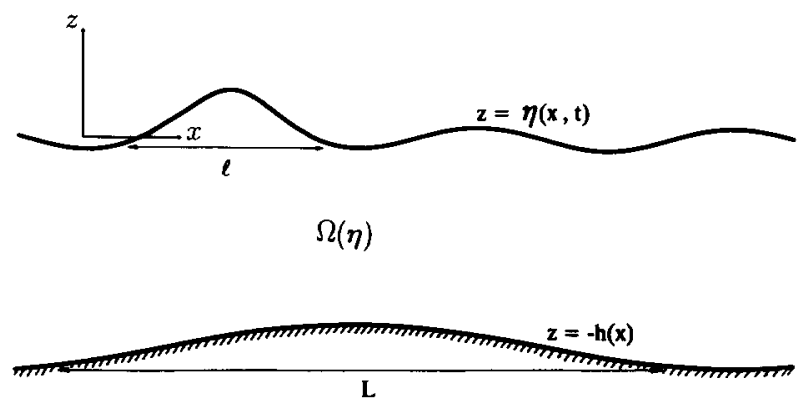

Fig. 1. The fluid domain.

with $P(\eta)$ and $K(\varphi, \eta)$ the potential energy and kinetic energy functional, respectively. Neglecting surface tension we have

$$
P(\eta)=\iint_{0}^{\eta} g z \mathrm{~d} z \mathrm{~d} x=\int \frac{1}{2} g \eta^{2} .
$$

The kinetic energy is given by

$$
K(\varphi, \eta)=\int_{\Omega(\eta)} \frac{1}{2} v^{2}=\int_{\Omega(\eta)} \frac{1}{2}|\nabla \Phi|^{2},
$$

with $\Phi$ the solution of the boundary value problem below

$$
\begin{aligned}
& \Delta \Phi=0, \quad \text { in } \Omega(\eta), \\
& \frac{\partial \Phi}{\partial \boldsymbol{n}}=0, \quad \text { at } \quad z=-h(x), \\
& \Phi(x, z, t)=\varphi(x, t), \quad \text { at } z=\eta(x, t) .
\end{aligned}
$$

The full set of equations describing the motion of the surface waves can be described as a Hamiltonian system (c.f. [1] and $[2,3]$ ) in the following way

$$
\partial_{t}\left(\begin{array}{l}
\varphi \\
\eta
\end{array}\right)=\left(\begin{array}{rr}
0 & -1 \\
1 & 0
\end{array}\right)\left(\begin{array}{l}
\delta_{\varphi} H \\
\delta_{\eta} H
\end{array}\right) .
$$

Here $\delta$ denotes the variational derivative with respect to the variables indicated. For the following it will be more convenient to introduce a new variable $u$ according to $u=\varphi_{x}$. Then eq. (17) becomes

$$
\partial_{t}\left(\begin{array}{l}
u \\
\eta
\end{array}\right)=\left(\begin{array}{cc}
0 & -\partial_{x} \\
-\partial_{x} & 0
\end{array}\right)\left(\begin{array}{l}
\delta_{u} H \\
\delta_{\eta} H
\end{array}\right)
$$

We look for simplified equations which have the same structure as eq. (18) by looking for a simpler Hamiltonian. Hence, the kinetic energy (15) will be approximated by an explicit expression in $\varphi$ and $\eta$. Looking for "rather long and rather low" waves over an even bottom, it will lead to Boussinesqtype of equations, describing waves running in both directions. By restricting to waves running in 
one direction only, it will lead to the $\mathrm{KdV}$ equation. In this paper we will consider in particular the modifications that take the effect of an uneven bottom into account.

\section{Approximations to Boussinesq equations}

The Boussinesq equations will be obtained by combining the approximation of dispersion and nonlinearity in a certain weighted way, to be specified precisely. To calculate the effect of dispersion and nonlinearity, the linear theory and the shallow water theory are considered first.

In linear theory, we approximate the kinetic energy $K(\varphi, \eta)$ by taking $\eta(x, t)=0$ in the boundary value problem (16). In the even bottom case, $h(x)=h_{0}, h_{0}$ is constant, eqs. (16) become

$$
\begin{aligned}
& \triangle \Phi=0, \quad \text { in } \quad \Omega(\eta), \\
& \frac{\partial \Phi}{\partial z}=0, \quad \text { at } \quad z=-h_{0}, \\
& \Phi(x, 0)=\varphi(x) .
\end{aligned}
$$

After some calculations one finds

$$
K(\varphi, \eta=0)=\int \frac{1}{2} \varphi_{x} \cdot R \varphi_{x}=\int \frac{1}{2} u \cdot R u,
$$

with $R$ a pseudo-differential operator with symbol

$$
\widehat{R}(k)=\frac{\tanh k h_{0}}{k}=h_{0}\left[1-\frac{1}{3}\left(k h_{0}\right)^{2}+\mathrm{O}\left(k h_{0}\right)^{4}\right] .
$$

We approximate the operator $R$ for long waves $(k \rightarrow 0)$ by the first two terms ${ }^{2}$ in the Taylor series for $\widehat{R}$, i.e. by

$$
h_{0}+\frac{1}{3} h_{0}^{3} \partial_{x}^{2}
$$

and we get

$$
K(u, 0)=\int \frac{1}{2} h_{0} u^{2}-\frac{1}{6} h_{0}^{3} u_{x}^{2}+\mathrm{O}\left(u_{x x}^{2}\right),
$$

as the approximation of the kinetic energy.

Different from the linear theory, in the tidal wave approximation it is assumed that the fluid particles have no vertical motion $\Phi(x, z, t)=\varphi(x, t)$ (ignoring dispersive effects). Then we get

$$
K(u, \eta)=\int \frac{1}{2} h_{0} u^{2}+\frac{1}{2} \eta u^{2}
$$

2 This approximation is simple but does not have the property that the operator is positive definite. A positive definite choice, as in [3], is possible and will influence the following only in an unessential way. For clarity of presentation we choose for the simpler approximation. 


\subsection{Boussinesq approximation}

Combining (20) and (21), we get as an approximation for the kinetic energy

$$
K(u, \eta)=\int \frac{1}{2} h_{0} u^{2}-\frac{1}{6} h_{0}^{3} u_{x}^{2}+\frac{1}{2} \eta u^{2}+\mathrm{O}\left(u_{x x}^{2}\right) .
$$

The typical Boussinesq equations are obtained with this expression under the assumption that the term accounting for dispersion and the term accounting for non-linearity are of the same order. To make that more precise, we introduce normalized variables as follows:

$$
\hat{\eta}=\eta / \epsilon, \quad \hat{u}=u / \epsilon, \quad \hat{x}=\sqrt{\epsilon} x, \quad \hat{t}=\sqrt{\epsilon} t,
$$

and consider $\hat{\eta}$ and $\hat{u}$ as functions of $\hat{t}$ and $\hat{x}$. That means that we consider surface elevations of the form

$$
\eta(x)=\epsilon \hat{\eta}(\sqrt{\epsilon} x)
$$

and the same for $u$. Introducing normalized variables in this way means that we consider waves which (if $\epsilon$ is sufficiently small) are low (amplitude $=O(\epsilon)$ ) and long (wavelength $\ell^{-1}=O\left(\epsilon^{1 / 2}\right)$ ), and which are such that both contributions in $K$ are of the same order. Then, for "rather long and rather low" waves we get as an approximation for the total energy

$$
H(u, \eta)=\epsilon^{3 / 2} \widehat{H}(\hat{u}, \hat{\eta}),
$$

where

$$
\widehat{H}(\hat{u}, \hat{\eta})=\int\left[\frac{1}{2} g \hat{\eta}^{2}+\frac{1}{2} h_{0} \hat{u}^{2}+\epsilon\left(-\frac{1}{6} h_{0}^{3} \hat{u}_{\hat{x}}^{2}+\frac{1}{2} \hat{\eta} \hat{u}^{2}\right)\right]+O\left(\epsilon^{2}\right)
$$

is the Hamiltonian in the normalized variables. Then eq. (18) reads in the normalized variables

$$
\partial_{\hat{i}}\left(\begin{array}{l}
\hat{u} \\
\hat{\eta}
\end{array}\right)=\left(\begin{array}{cc}
0 & -\partial_{\hat{x}} \\
-\partial_{\hat{x}} & 0
\end{array}\right)\left(\begin{array}{c}
\delta_{\hat{u}} \hat{H} \\
\delta_{\hat{\eta}} \hat{H}
\end{array}\right) .
$$

Substituting (22) in eq. (24) and omitting from now on the caps above the normalized variables, leads to the equations

$$
\begin{aligned}
& \partial_{t} \eta=-\partial_{x}\left[h_{0} u+\epsilon\left(\frac{1}{3} h_{0}^{3} u_{x x}+\eta u\right)\right]+O\left(\epsilon^{2}\right), \\
& \partial_{t} u=-\partial_{x}\left(g \eta+\frac{1}{2} \epsilon u^{2}\right)+O\left(\epsilon^{2}\right) .
\end{aligned}
$$

Omitting terms of order $\epsilon^{2}$ in eq. (25), we get one form of the well-known Boussinesq equations for surface waves. 


\subsection{Modification for varying bottom}

For the moment we return to physical, unscaled variables, and consider a slowly varying bottom for which the variations in $h$ occur over distances long compared to a wavelength. The reason will become clear in the following. To allow for enough flexibility in the presentation, we will consider functions $h$ of the form

$$
h(x)=\bar{h}\left(\epsilon^{\alpha+1 / 2} x\right)=\bar{h}\left(\epsilon^{\alpha} \hat{x}\right) \equiv \hat{h}(\hat{x}) .
$$

When $\alpha=0$ the changes in topography occur over the same distances as the waves we are looking for, but if $\alpha>0$ the changes in topography are slower. It turns out that the cases $\alpha<1$ and $\alpha \geqslant 1$ will have to be distinguished. For notational convenience let $\beta=\min \{1, \alpha\}$. Using the same approximations as in the even bottom case, i.e. linear theory and tidal wave approximation, we will find an approximation for $K(u, \eta)$.

In the linear theory, we take $\eta(x, t)=0$ in eq. (16). For (slowly) varying bottom we cannot solve this boundary value problem exactly. However, the assumption of slow variations allows one to replace the vanishing of the normal velocity at the bottom by the vanishing of the vertical component: $\partial \Phi / \partial z=0$ at $z=-h(x)$, and consider $h(x)$ as locally constant. Then one get just as before

$$
K(\varphi, \eta=0)=\int \frac{1}{2} \varphi_{x} \cdot R \varphi_{x}=\int \frac{1}{2} u \cdot R u,
$$

where now $R$ is a pseudo differential operator with symbol $\widehat{R}(k, x)=\tanh k h(x) / k$, which can be approximated like

$$
\widehat{R}(k, x)=h\left[1-\frac{1}{3}(k h)^{2}+\mathrm{O}\left((k h)^{4}\right)\right] .
$$

Symmetric differential operators that can be used to approximate the operator $R$ for long waves $(k \rightarrow 0)$ are

$$
h+\frac{1}{3} \partial_{x} h^{3} \partial_{x}, \quad h+\frac{1}{3} h \partial_{x} h \partial_{x} h, \quad h+\frac{1}{6}\left(h^{3} \partial_{x}^{2}+\partial_{x}^{2} h^{3}\right) .
$$

Each approximation is correct and equivalent up to order $\mathrm{O}\left(\epsilon^{1+\beta}\right)$ (if $\alpha \geqslant 1$ the terms that arise from $\mathrm{O}\left((k h)^{4}\right)$ in (27) are of order $\epsilon^{2}$ and will dominate). Choosing the first one of these, we get

$$
K(u, 0)=\int \frac{1}{2} h u^{2}-\frac{1}{6} h^{3} u_{x}^{2}+O\left(\epsilon^{1+\beta}\right) .
$$

Just like in the even bottom case, here we also use the tidal wave approximation to approximate the kinetic energy. We assume that $\Phi(x, z, t)=\varphi(x, t)$ (no dispersive effects), and we have as before

$$
K(u, \eta)=\int \frac{1}{2} h u^{2}+\frac{1}{2} \eta u^{2} .
$$

In the following we again use the normalized variables (22) introduced before. Then for "rather long and rather low waves" we get as the approximation for the total energy

$$
H(u, \eta)=\epsilon^{3 / 2} \widehat{H}(\hat{u}, \hat{\eta}),
$$


where now

$$
\widehat{H}(\hat{u}, \hat{\eta})=\int\left[\frac{1}{2} g \hat{\eta}^{2}+\frac{1}{2} \hat{h} \hat{u}^{2}+\epsilon\left(-\frac{1}{6} \hat{h}^{3} \hat{u}_{\hat{x}}^{2}+\frac{1}{2} \hat{\eta} \hat{u}^{2}\right)\right]+O\left(\epsilon^{1+\beta}\right) .
$$

is the Hamiltonian in the normalized variables. If we substitute (31) in eq. (24) and omit from now on the caps above the normalized variables, we find

$$
\begin{aligned}
& \partial_{t} \eta=-\partial_{x}\left[h u+\epsilon\left(\frac{1}{3} \partial_{x}\left(h^{3} u_{x}\right)+\eta u\right)\right]+\mathrm{O}\left(\epsilon^{1+\beta}\right), \\
& \partial_{t} u=-\partial_{x}\left(g \eta+\frac{1}{2} \epsilon u^{2}\right)+\mathrm{O}\left(\epsilon^{1+\beta}\right) .
\end{aligned}
$$

Omitting the lowest order terms $O\left(\epsilon^{1+\beta}\right)$, eqs. (32) are Boussinesq type equations for waves over uneven bottom.

\section{Uni-directionalization}

The Boussinesq equations (25) and (32) describe waves which run in two directions. To motivate the following, consider system (25). Omitting the Boussinesq effects all together by taking $\epsilon=0$, we have

$$
\partial_{t}\left(\begin{array}{l}
u \\
\eta
\end{array}\right)=\left(\begin{array}{rr}
0 & -\partial_{x} \\
-\partial_{x} & 0
\end{array}\right)\left(\begin{array}{l}
h_{0} u \\
g \eta
\end{array}\right) .
$$

By eliminating $u$ we have the standard second order wave equation for $\eta$

$$
\partial_{t}^{2} \eta=c_{0}^{2} \partial_{x}^{2} \eta
$$

with $c_{0}=\sqrt{g h_{0}}$. The solution of $(34)$ consists of waves which run to the right and to the left with speed $c_{0}$ : for arbitrary functions $F$ and $G$

$$
\eta(x, t)=F\left(x-c_{0} t\right)+G\left(x+c_{0} t\right) .
$$

We look for a transformation that makes it simpler to distinguish these uni-directional contributions in the full equations. We start with a transformation from $u, \eta$ to new variables $r$ and $s$. We look for these as a linear combination of $u$ and $\eta$ :

$$
\left(\begin{array}{l}
r \\
s
\end{array}\right)=A\left(\begin{array}{l}
u \\
\eta
\end{array}\right), \quad \text { with } A=\left(\begin{array}{ll}
a & b \\
c & d
\end{array}\right) .
$$

Substituting this transformation into eq. (24) and omitting all caps, we have

$$
\partial_{t}\left(\begin{array}{l}
r \\
s
\end{array}\right)=A\left(\begin{array}{rr}
0 & -\partial_{x} \\
-\partial_{x} & 0
\end{array}\right) A^{\mathrm{T}}\left(\begin{array}{l}
\delta_{r} H \\
\delta_{s} H
\end{array}\right) .
$$

For each choice of the matrix $A$ the structure map is given by

$$
A\left(\begin{array}{cr}
0 & -\partial_{x} \\
-\partial_{x} & 0
\end{array}\right) A^{\mathrm{T}}
$$


which is skew-symmetric. That implies that the Hamiltonian $H$ and hence the total energy is conserved. Also note that because the map is skew-symmetric, eq. (36) has the same (generalised) Hamiltonian structure as eq. (18). The transformation (35) which retains the Hamiltonian structure will be used for uni-directionalizing eq. (18) with a suitable choice for the matrix $A$. This will be done for even and uneven bottom separately.

\subsection{KdV equation for even bottom}

In the case of an even bottom, we choose for the transformation

$$
\eta=r-s, \quad u=\sqrt{\frac{g}{h_{0}}}(r+s) .
$$

or

$$
A=\frac{1}{2}\left(\begin{array}{cc}
\sqrt{\frac{h_{0}}{g}} & 1 \\
\sqrt{\frac{h_{0}}{g}} & -1
\end{array}\right) .
$$

Then with $H(u, \eta)=2 g \bar{H}(r, s)$, the Hamiltonian $\bar{H}$ as a functional of $r$ and $s$ is given by

$$
\bar{H}(r, s)=\int \frac{1}{2}\left(r^{2}+s^{2}\right)+\epsilon k(r, s)+\mathrm{O}\left(\epsilon^{2}\right),
$$

where

$$
k(r, s)=\int-\frac{1}{12} h_{0}^{2}\left(r_{x}+s_{x}\right)^{2}+\frac{1}{4 h_{0}}(r+s)^{2}(r-s) .
$$

Equation (36) become

$$
\partial_{t}\left(\begin{array}{l}
r \\
s
\end{array}\right)=\left(\begin{array}{cc}
-\Gamma_{0} & 0 \\
0 & \Gamma_{0}
\end{array}\right)\left(\begin{array}{l}
\delta_{r} \bar{H} \\
\delta_{s} \bar{H}
\end{array}\right),
$$

where $\Gamma_{0}=c_{0} \partial_{x}$ with $c_{0}=\sqrt{g h_{0}}$. Written in full the equations read:

$$
\begin{aligned}
& \partial_{t} r=-c_{0} \partial_{x}\left(r+\epsilon \delta_{r} k(r, s)\right)+\mathrm{O}\left(\epsilon^{2}\right), \\
& \partial_{t} s=c_{0} \partial_{x}\left(s+\epsilon \delta_{s} k(r, s)\right)+\mathrm{O}\left(\epsilon^{2}\right) .
\end{aligned}
$$

Concluding so far, the transformation (37) leads to the set of eqs. (39) which are completely equivalent to (25). The advantage of the formulation (39) is clear: in lowest order, taking $\epsilon=0$, we get equations for $r$ and $s$ which are uncoupled

$$
\partial_{t} r=-c_{0} \partial_{x} r, \quad \partial_{t} s=c_{0} \partial_{x} s .
$$

The $r$-equation describes right running waves, and the $s$-equation describes left running waves:

$$
r(x, t)=F\left(x-c_{0} t\right) \text { and } s(x, t)=G\left(x+c_{0} t\right) \text {, }
$$


respectively. This is the separation in lowest order that we have been looking for. For the complete eqs. (39) the $r$ and $s$ contribution are coupled, and no such simple splitting is possible. However, by restricting to a special set of initial data, we can show that a separation in the relevant order of the approximation is possible.

To that end, consider Boussinesq equations (39). We will look for solutions of these equations for which $r$ is of order 1, while $s$ is of order $\epsilon$. For such solutions we can replace in the equation for $r$ the term $\delta_{r} k(r, s)$ by

$$
\delta_{r} k(r, s)=\delta_{r} k(r, 0)+\mathrm{O}(\epsilon)
$$

and so $r$ satisfies in the same order of accuracy the equation

$$
\partial_{t} r=-c_{0} \partial_{x}\left(r+\epsilon \delta_{r} k(r)\right)+\mathrm{O}\left(\epsilon^{2}\right), \text { with } \bar{k}(r)=k(r, 0) .
$$

This is in fact the $\mathrm{KdV}$ equation. Writing it in a different way,

$$
\partial_{t} r=-c_{0} \partial_{x} \delta_{r} \bar{H}(r)+\mathrm{O}\left(\epsilon^{2}\right),
$$

where

$$
\bar{H}(r)=\int \frac{1}{2} r^{2}+\epsilon\left(-\frac{1}{12} h_{0}^{2} r_{x}^{2}+\frac{1}{4 h_{0}} r^{3}\right)
$$

In order to show that there are solutions for which $r=\mathrm{O}(1)$ and $s=\mathrm{O}(\epsilon)$, take an arbitrary initial value for $r$ :

$$
r_{0}(x)=f(x), \text { with } f=\mathrm{O}(1) .
$$

We use a perturbation argument in the following. The limit problem, putting $\epsilon=0$, shows that $r$ satisfies in lowest order $\partial_{t} r=-c_{0} \partial_{x} r$, with solution $r^{0}(x, t)=f\left(x-c_{0} t\right)$. Then $r-r^{0}=O(\epsilon)$. In the equation for $s$, the order $\epsilon$-term can be simplified:

$$
\delta_{s} k(r, s)=\delta_{s} k\left(r^{0}, 0\right)+\mathrm{O}\left(\epsilon, \epsilon^{\gamma}\right),
$$

if we assume a priori that $s=\mathrm{O}\left(\epsilon^{\gamma}\right),(\gamma>0)$. This shows that $s$ satisfies a linear equation, with order $\epsilon$-forcing

$$
\partial_{t} s=c_{0} \partial_{x}\left(s+\epsilon \delta_{s} k\left(r^{0}, 0\right)\right)+\mathrm{O}\left(\epsilon^{1+\gamma}, \epsilon^{2}\right) .
$$

The solution consists of a superposition of an arbitrary left-travelling wave and a particular solution $S$ which is a right-travelling wave:

$$
s(x, t)=g\left(x+c_{0} t\right)+S\left(x-c_{0} t\right) .
$$

Here $S=S(\xi)$ is given by

$$
2 S(\xi)+\epsilon \delta_{s} k(f(\xi), 0)=0,
$$

unique up to an unessential constant and clearly $S=\mathrm{O}(\epsilon)$. If $g$ is chosen to be of order $\epsilon$ or less, then $s=O(\epsilon)$ (and hence $\gamma=1$ ). This shows that solutions of the required order exist by choosing the initial data appropriately. (When we look for the uni-directional equation for the surface elevation in Section 5, it will be necessary to restrict to $g$ s that are of higher order, say $g=o(\epsilon)$.) 


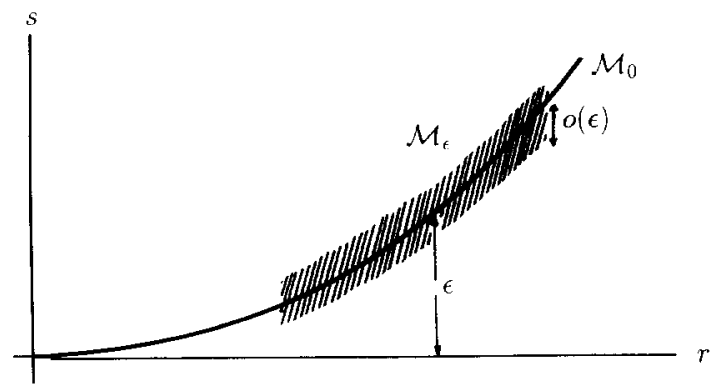

Fig. 2. The invariant manifold $\mathcal{M}_{0}$ and $\mathcal{M}_{\epsilon}$.

The procedure described above can be formulated in a more geometric way as follows. Define in $(r, s)$-function space the manifold

$$
\mathcal{M}_{0}=\left\{(r, s) \mid 2 s+\epsilon \delta_{s} k(r, 0)=0\right\} .
$$

Then, starting with initial data $\left(r_{0}, s_{0}\right) \in \mathcal{M}_{0}$ (for instance by taking $r_{0}$ arbitrary and determining $s_{0}$ appropriately), the resulting solution remains on $\mathcal{M}_{0}$. Therefore $s$ remains of order $\epsilon$, and the equation for $r$ is the $\mathrm{KdV}$ equation (43) in the required order $\epsilon^{2}$. If we allow $g$ in (45) to be non zero, but small, say $o(\epsilon)$, then the invariant manifold $\mathcal{M}_{0}$ is replaced by the thickened manifold

$$
\mathcal{M}_{\epsilon}=\left\{(r, s) \mid 2 s+\epsilon \delta_{s} k(r, 0)=o(\epsilon)\right\}
$$

see Fig. 2.

\subsection{Primary waves}

For uneven bottom we use the same transformation (37), but now with $h=h(x)$, a function of $x$ :

$$
\eta=r-s, \quad u=\sqrt{\frac{g}{h}}(r+s) .
$$

Just like for even bottom, we get for the Hamiltonian $H$ in the $r, s$ variables

$$
\bar{H}(r, s)=\int \frac{1}{2}\left(r^{2}+s^{2}\right)+\epsilon k(r, s)+\mathrm{O}\left(\epsilon^{1+\beta}\right)
$$

where

$$
k(r, s)=\int-\frac{1}{12} h^{2}\left(r_{x}+s_{x}\right)^{2}+\frac{1}{4 h}(r+s)^{2}(r-s),
$$

with $H(u, \eta)=2 g \bar{H}(r, s)$. Now, if we apply transformation (48), into eq. (36), it becomes

$$
\partial_{t}\left(\begin{array}{l}
r \\
s
\end{array}\right)=\left(\begin{array}{cc}
-\Gamma & -\frac{1}{2} c^{\prime} \\
\frac{1}{2} c^{\prime} & \Gamma
\end{array}\right)\left(\begin{array}{l}
\delta_{r} \bar{H} \\
\delta_{s} \bar{H}
\end{array}\right)
$$

with

$$
\Gamma=\frac{1}{2}\left(c(x) \partial_{x}+\partial_{x} c(x)\right) \text { and } c(x)=\sqrt{g h(x)}
$$


It must be noted that now the operator

$$
\left(\begin{array}{cc}
-\Gamma & -\frac{1}{2} c^{\prime} \\
\frac{1}{2} c^{\prime} & \Gamma
\end{array}\right)
$$

depends explicitly on the spatial variable $x$, and that the non-vanishing of the off-diagonal elements is a consequence of bottom variations. Nevertheless, the operator (52) is skew-symmetric, implying as before that the Hamiltonian $\bar{H}$ is constant and that eq. (50) has the same Hamiltonian structure as eq. (18). Equation (50) is written in full as

$$
\begin{aligned}
& \partial_{t} r=-\Gamma\left(r+\epsilon \delta_{r} k(r, s)\right)-\frac{1}{2} c^{\prime} \delta_{s} \bar{H}+\mathrm{O}\left(\epsilon^{1+\beta}\right), \\
& \partial_{t} s=\Gamma\left(s+\epsilon \delta_{s} k(r, s)\right)+\frac{1}{2} c^{\prime} \delta_{r} \bar{H}+\mathrm{O}\left(\epsilon^{1+\beta}\right) .
\end{aligned}
$$

Note that eqs. (53) and (32) are equivalent under the transformation (48), and that the equations are derived for bottom variations that satisfy (26) with $\alpha>0$.

In the rest of this section we first study the main (lowest order) contribution to the solution of (53). Therefore, first note that, neglecting Boussinesq effects by taking $\epsilon=0$, the equations for $r$ and $s$ are linearly coupled by the factor $c^{\prime}(x)$ :

$$
\begin{aligned}
& \partial_{t} r=-\Gamma r-\frac{1}{2} c^{\prime} s, \\
& \partial_{t} s=\Gamma s+\frac{1}{2} c^{\prime} r .
\end{aligned}
$$

To be able to take this coupling into account in the following, we will first study the characteristic property of the new operator $\Gamma(x)$. For the case of an even bottom, $\left(h(x)=h_{0}, h_{0}\right.$ constant), $\Gamma=c_{0} \partial_{x}$ and the $r$-equation is in lowest order $\partial_{t} r=-c_{0} \partial_{x} r$ with solutions $r(x, t)=r^{0}\left(x-c_{0} t\right)$, uniform travelling waves running to the right with velocity $c_{0}$. For the case of an uneven bottom, the $r$-equation of the Boussinesq equation (53) is in lowest order

$$
\partial_{t} r=-\Gamma(x) r, \text { with } \Gamma(x)=\frac{1}{2}\left(c(x) \partial_{x}+\partial_{x} c(x)\right) .
$$

The general solution of this equation can be written down explicitly:

$$
r(x, t)=\frac{1}{\sqrt{c(x)}} g(\sigma(x)-t), \text { with } \sigma^{\prime}(x)=\frac{1}{c(x)},
$$

and $g$ an arbitrary function. The function $g$ in $(56)$ is related to the initial data $r(x, 0)=r^{0}(x)$ according to

$$
r^{0}(x)=\frac{g(\sigma(x))}{\sqrt{c(x)}} .
$$

The solution (56) expresses the fact that the value of $g$ is constant on the characteristic $\mathrm{d} x / \mathrm{d} t=c(x)$ and that $r$ is obtained by an additional multiplication with $1 / \sqrt{c(x)}$, see Fig. 3. Stated differently,

$$
\sqrt{c(x)} r(x, t)=\sqrt{c\left(x_{0}\right)} r^{0}\left(x_{0}\right)=\text { constant }
$$




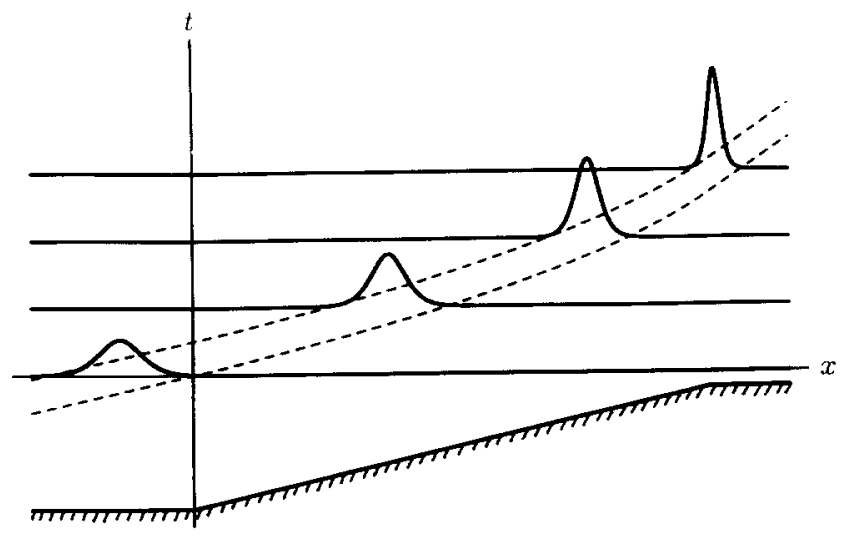

Fig. 3. Distorted solitary waves over an uneven bottom described by (56) on the characteristic curve $\mathrm{d} x / \mathrm{d} t=c(x)$.

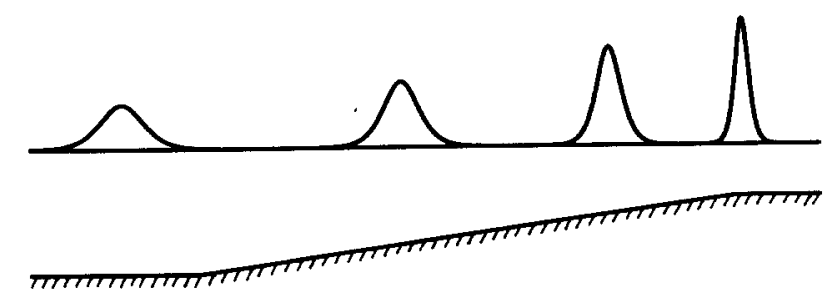

Fig. 4. Wave profiles at different times $t_{1}<t_{2}<t_{3}<t_{4}$, indicating amplitude increase and wavelength decrease for decreasing depth.

on the characteristic $\mathrm{d} x / \mathrm{d} t=c(x)$ which passes through $\left(x_{0}, t=0\right)$. From this explicit solution, it can be observed that the wave amplitude is proportional to $1 / \sqrt{c(x)}$ and that the wave velocity is $c(x)$. The change in the wavelength $\ell(x)$ (see Fig. 3), follows from the approximation

$$
\frac{\ell^{\prime}(x)}{\ell(x)} \simeq \frac{c^{\prime}(x)}{c(x)} .
$$

Hence, if $c(x)$ decreases, the wave amplitude increases and the wave velocity and the wavelength decrease, see Fig. 4. From this we may conclude that already in this first approximation, eq. (55) shows the characteristic features of a wave during run up. This is all attributed to the specific form of the map $\Gamma(x)$.

For the linear equation (55) with an inhomogeneous term added (such as in eq. (54)), the explicit solution can still be obtained. Consider the general equation

$$
\partial_{t} r=-\Gamma(x) r+b, \quad r(x, 0)=r_{0}(x),
$$

with some given function $b=b(x, t)$ as source and $r_{0}$ as initial condition. For a more efficient notation we denote the solution of the initial value problem

$$
\partial_{t} r=-\Gamma(x) r, \quad r(x, 0)=r_{0}(x),
$$


by

$$
r(x, t)=\Phi_{t}\left(r_{0}\right)(x),
$$

so that $\Phi_{t}$ denotes the 'flow', mapping the initial data to the solution after time $t$. (An explicit, rather unpleasant, expression for $\Phi_{t}$ follows from (56), (57).) Then the solution of the inhomogeneous problem (58) is given by the Duhamel integral (variation of constant formula)

$$
r(x, t)=\Phi_{t}\left(r_{0}\right)(x)+\int_{0}^{t} \Phi_{t-\tau} b(x, \tau) \mathrm{d} \tau .
$$

\section{3. $A K d V$-type of equation for uneven bottom}

In much the same way as for the even bottom, we now consider the uni-directionalization for a varying bottom for which $c^{\prime}=\mathrm{O}\left(\epsilon^{\alpha}\right)$, with $\alpha>0$. Consider the Boussinesq equations (53) and observe that in the same order of truncation, the terms with $c^{\prime}$ can be simplified. Then the equations read:

$$
\begin{aligned}
& \partial_{t} r=-\Gamma(x)\left(r+\epsilon \delta_{r} k(r, s)\right)-\frac{1}{2} c^{\prime} s+\mathrm{O}\left(\epsilon^{1+\beta}\right), \\
& \partial_{t} s=\Gamma(x)\left(s+\epsilon \delta_{s} k(r, s)\right)+\frac{1}{2} c^{\prime} r+\mathrm{O}\left(\epsilon^{1+\beta}\right) .
\end{aligned}
$$

For a solution with $r=\mathrm{O}(1)$, we will show that there exists a solution $s=\mathrm{O}\left(\epsilon^{\beta}\right)$ for appropriate initial data for $s$. (Recall $\beta=\min \{1, \alpha\}$ ). For such solutions, in the $r$-equation $\delta_{r} k(r, s)$ can be replaced by $\delta_{r} k(r, 0)$ and we get

$$
\partial_{t} r=-\Gamma\left(r+\epsilon \delta_{r} \bar{k}(r)\right)-\frac{1}{2} c^{\prime} s+\mathrm{O}\left(\epsilon^{1+\beta}\right), \text { with } \quad k(r)=k(r, 0),
$$

or equivalently,

$$
\partial_{t} r=-\Gamma \delta_{r} \bar{H}(r)-\frac{1}{2} c^{\prime} s+\mathrm{O}\left(\epsilon^{1+\beta}\right), \quad \text { with } \bar{H}(r)=\int \frac{1}{2} r^{2}+\epsilon \bar{k}(r) .
$$

Since $c^{\prime} s=O\left(\epsilon^{\alpha+\beta}\right)$, two cases can be considered.

If $\alpha \geqslant 1$, which means very mild bottom variations, then $\beta=1$ and $c^{\prime} s=O\left(\epsilon^{1+\alpha}\right)$ is of the same order as the error in the equation and can therefore be neglected. Then $r$ satisfies a KdV-type of equation without forcing:

$$
\partial_{t} r=-\Gamma\left(r+\epsilon \delta_{r} \bar{k}\right)+\mathrm{O}\left(\epsilon^{2}\right) .
$$

This equation is just the standard KdV equation (43) when the bottom is flat. Else, it is a generalization of the $\mathrm{KdV}$ with coefficients that depend explicitly on the topography.

If $\alpha<1$, then $\beta=\alpha$ and the term $c^{\prime} s=\mathrm{O}\left(\epsilon^{2 \alpha}\right)$ will dominate the truncation error in the equation. Hence, $r$ satisfies a $\mathrm{KdV}$ equation with forcing:

$$
\partial_{t} r=-\Gamma\left(r+\epsilon \delta_{r} \bar{k}(r)\right)-\frac{1}{2} c^{\prime} s+\mathrm{O}\left(\epsilon^{1+\alpha}\right) .
$$

The forcing term $\frac{1}{2} c^{\prime} s$ accounts for the unevenness and depends on the reflected wave $s\left(=O\left(\epsilon^{\alpha}\right)\right)$. This reflected wave $s$ will be given explicitly in Section 5.2 in terms of the initial data for $r$. 
To show the existence of solutions for which $r=\mathrm{O}(1)$ and $s=\mathrm{O}\left(\epsilon^{\beta}\right)$, we take an arbitrary initial data $r_{0}(x)=\mathrm{O}(1)$ and let $r^{0}(x, t)$ be the solution of the limit problem (see Section 4.2). Assuming a priori that $s=\mathrm{O}\left(\epsilon^{\gamma}\right)$ and since $r-r^{0}=\mathrm{O}\left(\epsilon^{\beta}\right)$ we can simplify

$$
\delta_{s} k(r, s)=\delta_{s} k\left(r^{0}, 0\right)+\mathrm{O}\left(\epsilon^{\beta}, \epsilon^{\gamma}\right) \text { and } \frac{1}{2} c^{\prime} r=\frac{1}{2} c^{\prime} r^{0}+\mathrm{O}\left(\epsilon^{\alpha+\beta}\right) .
$$

Then $s$ satisfies

$$
\partial_{t} s=\Gamma s+\epsilon \Gamma \delta_{s} k\left(r^{0}, 0\right)+\frac{1}{2} c^{\prime} r^{0}+\mathrm{O}\left(\epsilon^{1+\beta}, \epsilon^{1+\gamma}, \epsilon^{\alpha+\beta}\right) .
$$

This is a linear equation for $s$, with inhomogeneous terms of order $O(\epsilon)$ and $O\left(\epsilon^{\alpha}\right)$ respectively. The solution can be written like

$$
s(x, t)=S_{0}(x, t)+S_{1}(x, t)+S_{2}(x, t),
$$

where $S_{0}$ is an arbitrary solution of the homogeneous equation $\partial_{t} s=\Gamma s$ and $S_{1}=\mathrm{O}\left(\epsilon^{\alpha}\right)$ and $S_{2}=\mathrm{O}(\epsilon)$ are particular solutions accounting for the two inhomogeneous terms. Choosing initial data for which $S_{0}=0$, the solution $s$ is of the required order: $s=\mathrm{O}\left(\epsilon^{\beta}\right)$ (and hence, $\gamma=\beta$ ). In Section 5.2 we will give explicit (approximate) expressions for $S_{1}$ and $S_{2}$.

\section{Equations for the surface elevation}

In the foregoing section we obtained KdV-type of eqs. (43) and (62) in $r$ for suitable initial data, both in case of an even and in case of an uneven bottom. This variable $r$ is not the surface elevation itself. Since $\eta=r-s$, and $s$ is larger than the residual in the equation, a further elaboration is required to derive the equation for $\eta$ itself. In this section we will derive the equation for the surface elevation $\eta$ under the assumption that the wave is uni-directional, explicitly $r=\mathrm{O}(1)$ and $s$ is small. First we deal with an even bottom and then we turn to uneven bottom.

\subsection{Even bottom}

Consider the standard $\mathrm{KdV}$ equation (43) in the $r$ variable for an even bottom. The equation for $\eta=r-s$, with $r=\mathrm{O}(1)$ and $s=\mathrm{O}(\epsilon)$, is given by

$$
\partial_{t}(r-s)=-c_{0} \partial_{x}\left((r-s)+\epsilon \delta_{r} k(r, 0)\right)-\left[\partial_{t} s+c_{0} \partial_{x} s\right]+\mathrm{O}\left(\epsilon^{2}\right) .
$$

The term $\delta_{r} k(r, 0)$ can be approximated by $\delta_{\eta} \bar{k}(\eta)$, where $\bar{k}(\eta) \equiv k(\eta=r-s, 0)$, correct up to $\mathrm{O}(\epsilon)$. Therefore the wave elevation $\eta$ evolves according to a $\mathrm{KdV}$ equation with forcing,

$$
\partial_{t} \eta=-c_{0} \partial_{x} \delta_{\eta} \bar{H}(\eta)-\mathcal{F}+\mathrm{O}\left(\epsilon^{2}\right)
$$

with

$$
\bar{H}(\eta)=\int \frac{1}{2} \eta^{2}+\epsilon \bar{k}(\eta) \text { and } \mathcal{F}=\partial_{t} s+c_{0} \partial_{x} s
$$

The expression of the forcing shows that the forcing consists solely of contributions from waves running to the left. For initial data on the manifold $\mathcal{M}_{0}$ given by (46), this forcing is zero. Then we 
have that the surface elevation $\eta$ evolves according to the well-known standard KdV equation without forcing

$$
\partial_{t} \eta=-c_{0} \partial_{x} \delta_{\eta} \bar{H}(\eta)+\mathrm{O}\left(\epsilon^{2}\right) .
$$

For $(r, s)$ in the thickened manifold $\mathcal{M}_{\epsilon}$ given by (47), the order of the forcing $\mathcal{F}$ is exactly the order of $g$ in (45). And so, $\mathcal{F}=o(\epsilon)$. One could say that restriction to the thickened manifold $\mathcal{M}_{\epsilon}$, implicitly defines what is meant by restriction to waves running mainly in one direction.

The standard $\mathrm{KdV}$ equation (65) is well-known to be completely integrable. It has infinitely many conserved integrals. For later reference, we recall the simplest three of these: the total energy functional

$$
H(\eta)=\int \frac{1}{2} \eta^{2}+\epsilon\left(-\frac{1}{12} h_{0}^{2} \eta_{x}^{2}+\frac{1}{4 h_{0}} \eta^{3}\right)
$$

the momentum functional

$$
M(\eta)=\int \frac{\eta^{2}}{2 c_{0}}
$$

and the mass-like functional

$$
C(\eta)=\int \frac{\eta}{\sqrt{c_{0}}}
$$

\subsection{Uneven bottom}

Just as in the case of a constant bottom, we will look for an equation for the surface elevation $\eta$ itself. Consider the KdV-type of equation in the $r$ variable for uneven bottom, eq. (60). The evolution of $\eta=r-s$, with $r=\mathrm{O}(1), s=\mathrm{O}\left(\epsilon^{\beta}\right)$ is described by

$$
\partial_{t}(r-s)=-\Gamma\left((r-s)+\epsilon \delta_{r} k(r, 0)\right)-\left[\partial_{t} s+\Gamma(x) s+\frac{1}{2} c^{\prime} s\right]+\mathrm{O}\left(\epsilon^{1+\beta}\right) .
$$

The term in $\delta_{r} k(r, 0)$ can be approximated by $\delta_{\eta} \bar{k}(\eta)$, with $k(\eta) \equiv k(\eta=r-s, 0)$, correct up to order $\mathrm{O}\left(\epsilon^{\beta}\right)$. Hence, the surface elevation $\eta$ evolves according to the KdV-type of equation with forcing

$$
\partial_{t} \eta=-\Gamma(x) \delta_{\eta} \bar{H}(\eta)-\mathcal{F}+\mathrm{O}\left(\epsilon^{1+\beta}\right),
$$

with

$$
\bar{H}(\eta)=\int \frac{1}{2} \eta^{2}+\epsilon \bar{k}(\eta) \text { and } \mathcal{F}=\partial_{t} s+\Gamma s+\frac{1}{2} c^{\prime} s
$$

The term $\frac{1}{2} c^{\prime} s$ in the forcing $\mathcal{F}$ accounts for the contribution from the unevenness, while $\partial_{t} s+\Gamma s$ would vanish for a right running wave as the primary wave considered before. To investigate this forcing, we consider the equation to be satisfied by $s$ more closely. This equation reads:

$$
\partial_{t} s=\Gamma\left(s+\epsilon \delta_{s} k\left(r^{0}, 0\right)\right)+\frac{1}{2} c^{\prime} r^{0}+O\left(\epsilon^{2 \beta}\right) .
$$

We will look for an approximate solution (in the required order) as the sum $s=S_{1}+S_{2}$, where $S_{1}$ is the solution of

$$
\partial_{t} s=\Gamma s+\frac{1}{2} c^{\prime} r^{0}, \quad \text { with } \quad S_{1}=\mathrm{O}\left(\epsilon^{\alpha}\right)
$$


and $S_{2}$ is the solution of

$$
\partial_{t} s=\Gamma\left(s+\epsilon \delta_{s} k\left(r^{0}, 0\right)\right), \quad \text { with } \quad S_{2}=\mathrm{O}(\epsilon) .
$$

The forcing $\mathcal{F}$ can be split accordingly

$$
\mathcal{F}=\mathcal{F}_{1}+\mathcal{F}_{2}, \quad \text { where } \quad \mathcal{F}_{1,2}=\partial_{t} S_{1,2}+\Gamma S_{1,2}+\frac{1}{2} c^{\prime} S_{1,2}
$$

An approximate solution for $S_{1}$ which is correct up to order $O\left(\epsilon^{2 \alpha}\right)$ is given by

$$
S_{1}=-\frac{c^{\prime}(x)}{4 \sqrt{c(x)}} F(\sigma(x)-t)
$$

where $F$ is a primitive of $\sqrt{c(x)} r_{0}(x)$. Indeed, for this function it holds that

$$
\partial_{t} s-\Gamma s-\frac{1}{2} c^{\prime}(x) r^{0}=\frac{1}{4} \sqrt{c(x)} c^{\prime \prime}(x) F(\sigma(x)-t),
$$

which is of the order $c^{\prime \prime}(x)=\mathrm{O}\left(\epsilon^{2 \alpha}\right)$. For this solution, the forcing $\mathcal{F}_{1}$ in the equation for $\eta$ is given by

$$
\mathcal{F}_{1}=-\frac{1}{4} \partial_{x}\left(c^{\prime} \sqrt{c}\right) F(\sigma(x)-t)+\mathrm{O}\left(\epsilon^{3 \alpha}\right) .
$$

From this we conclude that $\mathcal{F}_{1}$ is of order $O\left(\epsilon^{2 \alpha}\right)$.

An approximate solution for $S_{2}$ is given by

$$
S_{2}=-\frac{\epsilon}{12} \frac{c^{3 / 2}}{g^{2}} f^{\prime \prime}(\sigma(x)-t)-\frac{\epsilon g}{8 c^{3}} f^{2}(\sigma(x)-t) .
$$

After some calculations it is found that $S_{2}$ satisfies the equation in the required order:

$$
\partial_{t} s-\Gamma s-\epsilon \Gamma \delta_{s} k\left(r^{0}, 0\right)=\mathrm{O}\left(\epsilon^{1+\alpha}\right)
$$

and one obtains $\mathcal{F}_{2}=\mathrm{O}\left(\epsilon^{1+\alpha}\right)$. Hence, the total forcing $\mathcal{F}$ is of the order

$$
\mathcal{F}=\mathcal{F}_{1}+\mathcal{F}_{2}=\mathrm{O}\left(\epsilon^{2 \alpha}\right)+\mathrm{O}\left(\epsilon^{1+\alpha}\right) .
$$

Since the error in eq. (69) is $O\left(\epsilon^{1+\beta}\right)$, the forcing $\mathcal{F}_{2}=O\left(\epsilon^{1+\alpha}\right)$ can be neglected, and $\mathcal{F}$ in $(69)$ is only $\mathcal{F}_{1}$.

The dependence of $\mathcal{F}$ on $\eta$ can be made explicit, since the initial data for $\eta$ and for $r$ only differ by $s_{0}(x)=\mathrm{O}\left(\epsilon^{\beta}\right)$. Both initial data will give the same forcing $\mathcal{F}$ in the following approximation, i.e.

$$
\mathcal{F}=-\frac{1}{4} \partial_{x}\left(c^{\prime} \sqrt{c}\right) F(\sigma(x)-t)+\mathrm{O}\left(\epsilon^{2 \alpha+\beta}\right) .
$$

Here $F(y)$ is a primitive of the function $f(y)$ that follows from the initial data:

$$
\eta_{0}(x)=\frac{1}{\sqrt{c(x)}} f(\sigma(x)) .
$$

Therefore, we get the KdV-type of equation with explicit forcing,

$$
\partial_{t} \eta=-\Gamma(x) \delta_{\eta} \bar{H}(\eta)+\frac{1}{4} \partial_{x}\left(c^{\prime} \sqrt{c}\right) F(\sigma(x)-t)+\mathrm{O}\left(\epsilon^{2 \alpha+\beta}, \epsilon^{1+\beta}\right) .
$$


Three cases, dealing with various types of bottom variations can be distinguished.

(i) For $\alpha \geqslant 1$ (then $\beta=1$ ), which means very mild bottom variations, the forcing is of higher order than the accuracy of the equation and can therefore be neglected. Then the surface elevation $\eta$ evolves according to the $\mathrm{KdV}$-type of equation without forcing

$$
\partial_{t} \eta=-\Gamma(x) \delta_{\eta} \bar{H}(\eta)+\mathrm{O}\left(\epsilon^{2}\right)
$$

Note that this agrees with the limiting case of an even bottom for which (74) reduces to the standard $\mathrm{KdV}$ equation (65).

(ii) For $0<\alpha<1$ (then $\beta=\alpha$ ), the order of the forcing $O\left(\epsilon^{2 \alpha}\right)$ cannot be neglected compared to the error $O\left(\epsilon^{1+\alpha}\right)$ in the equation.

(a) If $\frac{1}{2} \leqslant \alpha<1$, the order of forcing is essential, but smaller than the Boussinesq effects that are $O(\epsilon)$, and we obtain $\mathrm{KdV}$-type of equation with forcing

$$
\partial_{t} \eta=-\Gamma(x) \delta_{\eta} \bar{H}(\eta)+\frac{1}{4} \partial_{x}\left(c^{\prime} \sqrt{c}\right) F(\sigma(x)-t)+\mathrm{O}\left(\epsilon^{1+\alpha}\right)
$$

(b) If $0<\alpha<\frac{1}{2}$, the forcing dominates the Boussinesq terms that are of $O(\epsilon)$. Then up to $O(\epsilon)$, the equation for $\eta$ is linear with forcing

$$
\partial_{t} \eta=-\Gamma \eta+\frac{1}{4} \partial_{x}\left(c^{\prime} \sqrt{c}\right) F(\sigma(x)-t)+\mathrm{O}(\epsilon)
$$

the explicit solution will be discussed in the next subsection.

A final note about the (unforced) KdV-type of eq. (74). Since the coefficients depend explicitly on $x$, this equation will (most probably) not be completely integrable. Nevertheless, the Hamiltonian structure implies that the energy $H$ is conserved:

$$
H(\eta)=\int \frac{1}{2} \eta^{2}+\epsilon\left(-\frac{1}{12} h^{2} \eta_{x}^{2}+\frac{1}{4 h} \eta^{3}\right)
$$

because $\Gamma$ is skew-symmetric. Since $1 / \sqrt{c(x)}$ is in the kernel of $\Gamma$, there is another conserved quantity, a mass-like functional:

$$
C(\eta)=\int \frac{\eta}{\sqrt{c(x)}} .
$$

The momentum functional

$$
M(\eta)=\int \frac{\eta^{2}}{2 c(x)},
$$

which is conserved for the even bottom case because of translation symmetry, is no longer conserved in the case of uneven bottom. 


\subsection{Causality}

Consider the linear equation (76) which is obtained for $0<\alpha<\frac{1}{2}$. We discussed already the explicit solution of the homogeneous part which is called the primary wave. An approximate solution for the full equation (76) is given by

$$
\eta(x, t)=\frac{1}{\sqrt{c(x)}} f(\sigma(x)-t)+\frac{\beta(x)}{\sqrt{c(x)}} F(\sigma(x)-t)+\mathrm{O}\left(\epsilon^{\alpha}\right),
$$

where

$$
\frac{\mathrm{d} \beta}{\mathrm{d} x}=\frac{\partial_{x}\left(c^{\prime} \sqrt{c}\right)}{4 \sqrt{c}}
$$

and $F$ is a primitive of $f$. We call the second term of $(80)$ the "secondary wave".

Note that in this solution and in (75) and (76) the primitive $F$ of $f$ appears. Clearly $F$ is defined only up to a constant. In the following, we will use a causality argument to show that a unique $F$ can be defined. To that end it is most appealing to consider an initial wave with bounded support, say

$$
\eta_{0}(x)=0, \text { for } x<x_{-} \text {and for } x>x_{+}
$$

and a localized variation in the bottom, say

$$
c(x)=c_{-}, \text {for } x<a \text { and } c(x)=c_{+}, \text {for } x>b .
$$

The localized bottom variations will cause the forcing $\mathcal{F}$ to be zero outside the unevenness (since there $\left.c^{\prime}=0\right)$ :

$$
\mathcal{F}=0, \text { for } x<a \text { and for } x>b .
$$

Assuming that the initial wave is upstream of the bottom variations, i.e. $x_{+}<a$, see Fig. 5 , it is natural to assume that the forcing $\mathcal{F}$ is zero until the wave arrives at the unevenness. This leads one to define uniquely the function $F(\sigma)$ such that $F(\sigma)=0$ for $\sigma>\sigma\left(x_{+}\right)$, i.e. to define

$$
F(\sigma)=-\int_{\sigma}^{\sigma\left(x_{+}\right)} f(\hat{\sigma}) \mathrm{d} \hat{\sigma} .
$$

To investigate what happens after the wave has passed the obstacle, note that

$$
F(\sigma)=\text { constant }=-\int_{\sigma\left(x_{-}\right)}^{\sigma\left(x_{+}\right)} f(\hat{\sigma}) \mathrm{d} \hat{\sigma} \equiv \mathcal{R}, \text { for } \sigma<\sigma\left(x_{-}\right),
$$

see also Fig. 5. This implies that, whenever $\mathcal{R}$ is nonzero, the forcing will remain to be present above the bottom variations, after the lowest order contribution of the wave has passed the unevenness, see Fig. 6. This remaining force is constant in time and given by

$$
\mathcal{F}=-\frac{1}{4} \mathcal{R} \partial_{x}\left(\sqrt{c(x)} c^{\prime}(x)\right)
$$




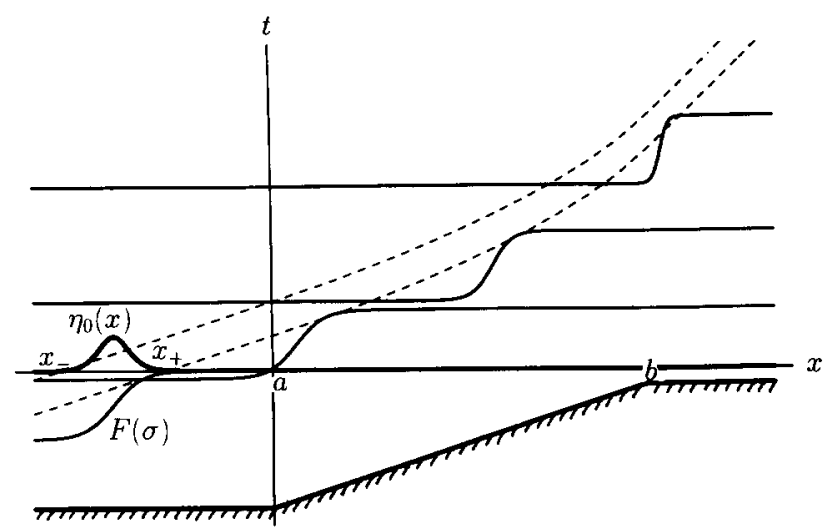

Fig. 5. The bounded support initial wave which is downstream of the localized variation in the bottom and the unique function $F(\sigma)$ as a primitive of $f(\sigma)$.

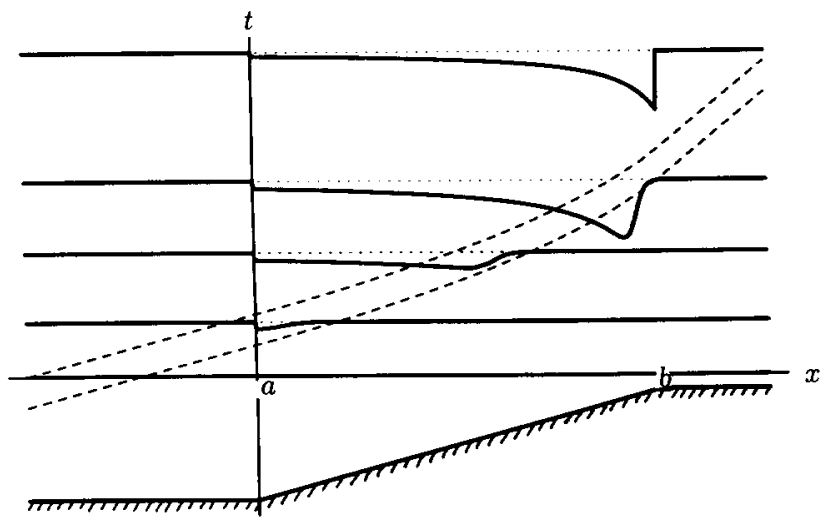

Fig. 6. The forcing $\mathcal{F}$ as a function of time.

After the wave has passed the unevenness, the remaining solution is given by (c.f. (80))

$$
\eta(x, t)=\frac{\beta(x)}{\sqrt{c(x)}} \mathcal{R} .
$$

By specifying the constant in the function $\beta$, which function is determined only up to a constant by $(81)$, it is possible to have a vanishing wave upstream. Therefore, let $\beta(x)=0$ for $x<a$, by defining

$$
\beta(x)=\frac{1}{4} \int_{a}^{x} \frac{\left(c^{\prime} \sqrt{c}\right)^{\prime}}{\sqrt{c}}
$$

Then, just like the forcing $\mathcal{F}$ in case $\mathcal{R} \neq 0$, the secondary wave remains above the unevenness after the wave has passed. This remaining wave may be recognized as a "tail wave". This tail wave is of higher order $\mathrm{O}\left(\left(c^{\prime}\right)^{2}\right)$. 
Having exploited the causality principle in this way, we may generalize to incorporate non-confined initial profiles in a formal manner and define the forcing in an unique way by taking

$$
F(\sigma)=-\int_{\sigma}^{\infty} f(\hat{\sigma}) \mathrm{d} \hat{\sigma} .
$$

Then the longterm effect (for $t \rightarrow \infty$ ) is measured again by

$$
\mathcal{R} \equiv-\int_{-\infty}^{\infty} f(\hat{\sigma}) \mathrm{d} \hat{\sigma} .
$$

A final remark is that the value of this quantity can be expressed directly in terms of the initial profile $\eta_{0}$, both in case of localized and in case of non-localized bottom variations:

$$
\begin{aligned}
\mathcal{R} & \equiv-\int_{-\infty}^{\infty} f(\hat{\sigma}) \mathrm{d} \hat{\sigma}=-\int_{-\infty}^{\infty} \sqrt{c(x)} \eta_{0}(x) \sigma^{\prime}(x) \mathrm{d} x \\
& =-\int_{-\infty}^{\infty} \frac{1}{\sqrt{c(x)}} \eta_{0}(x) \mathrm{d} x=-C\left(\eta_{0}\right) .
\end{aligned}
$$

We recognize here $C(\eta)$ as the mass-like functional (78) for the KdV-type of eq. (74).

\section{Comparison with Newell's result}

In the literature several other equations can be found that describe uni-directional waves above topography (see the next section for some references). The way to derive these equations often involves many transformations, and the use of variables that are different from a physical quantity like the elevation. These facts often obscure the assumptions that are made in the derivation.

In this section we will show how one particular case considered in this paper can be compared, and agrees with a result derived by Newell [8]. To make a comparison easier, we present a brief outline of the method described by Newell. This also shows clearly the difference in the derivation between the Hamiltonian approach in this paper and the series-expansion method that is usually employed.

Newell uses scaled variables in much the same way as in (22) with $g=1$. The bottom variations are required to satisfy $h(x)=\hat{h}(\epsilon \hat{x})$, so Newell considers the case $\alpha=1$. In the scaled variables, the full basic equations read:

$$
\begin{aligned}
& \epsilon \Phi_{x x}+\Phi_{z z}=0, \quad \text { in } \Omega(\eta), \\
& \epsilon^{2} \Phi_{x} h_{x}+\Phi_{z}=0, \text { at } z=-h(x), \\
& \Phi_{t}+\eta+\frac{1}{2} \epsilon \Phi_{x}^{2}+\frac{1}{2} \Phi_{z}^{2}=0, \quad \text { at } z=\epsilon \eta \\
& \eta_{t}+\epsilon \Phi_{x} \eta_{x}=\frac{1}{\epsilon} \Phi_{z}, \quad \text { at } z=\epsilon \eta
\end{aligned}
$$


Then a power-series solution in $z$ is taken that satisfies the no-flux condition through the bottom and the Laplace equation:

$$
\begin{aligned}
\Phi(x, z, t)= & F(x, t)-\frac{\epsilon}{2} F_{x x}(x, t)(z+h)^{2} \\
& +\epsilon^{2}\left(\frac{1}{24} F_{x x x x}(z+h)^{4}-h_{x} F_{x}(x, t)(z+h)\right)+O\left(\epsilon^{3}\right) .
\end{aligned}
$$

Introducing $w \equiv F_{x}$, the free boundary conditions in (83) lead to the following set of Boussinesq-type of equations:

$$
\begin{aligned}
& w_{t}+\eta_{x}=\epsilon\left(\frac{1}{2} h^{2} w_{x x t}-w w_{x}\right)+\mathrm{O}\left(\epsilon^{2}\right), \\
& \eta_{t}+(h w)_{x}=\epsilon\left(\frac{1}{6} h^{3} w_{x x x}-(\eta w)_{x}\right)+\mathrm{O}\left(\epsilon^{2}\right) .
\end{aligned}
$$

These equations are different from the Boussinesq equations given in (32) for $\alpha=1$. This can be attributed to another choice for the effect of dispersion and another meaning of the variables $u$ and $w$. Nevertheless, the equations are equivalent in the required order. For instance, for $h=1$, the dispersion relation for $(85)$ :

$$
\omega(k)=k\left(1-\frac{\epsilon}{3} k^{2}\right)^{1 / 2}
$$

agrees in the required order with the choice (28) for the pseudo differential operator $R$ made in this paper. (It may also be observed that, different from (32), eqs. (85) do not have a Hamiltonian structure.)

Introducing characteristic coordinates

$$
\boldsymbol{\theta}_{ \pm}=\sigma(x) \mp t, \text { with } \frac{\mathrm{d} \sigma}{\mathrm{d} x}=\frac{1}{c(x)}
$$

solutions are sought for which the right travelling effects dominate. Writing $\boldsymbol{\theta}_{+}=\boldsymbol{\theta}$, and

$$
F(x, t)=f(\boldsymbol{\theta}, \epsilon X)+\mathrm{O}(\epsilon), \quad q=\frac{3}{2} h^{-2} f_{\boldsymbol{\theta}}, \quad \tau=\frac{1}{6} \int^{X} h^{1 / 2} \mathrm{~d} x,
$$

Newell finds the following equation:

$$
q_{\tau}+6 q q_{\theta}+q_{\theta \theta \theta}=-\Pi q, \text { with } \Pi=\frac{9 h_{\tau}}{4 h} .
$$

This equation turns out to possess two conserved functionals:

$$
\int h^{9 / 4} q \mathrm{~d} \boldsymbol{\theta} \text { and } \int h^{9 / 2} q^{2} \mathrm{~d} \boldsymbol{\theta}
$$

The variable $q$ as introduced by Newell has no immediate physical meaning. However, it can be shown, by using the Boussinesq equation (85), that $q$ is related in a simple way to the wave elevation:

$$
\eta(\theta, \tau)=\frac{2}{3} h^{2} q+\mathrm{O}(\epsilon)
$$


(so, in fact, $\eta=f_{\theta}+\mathrm{O}(\epsilon)$ ). Expressed in $\eta$, eq. (88) transforms to

$$
\eta_{\tau}+9 h^{-2} \eta \eta_{\theta}+\eta_{\theta \theta \theta}=-\frac{1}{9} \Pi \eta
$$

and the conserved integrals (89) become:

$$
\frac{3}{2} \int \frac{\eta}{\sqrt{c(x)}} \mathrm{d} x \text { and } \frac{9}{4} \int \eta^{2} \mathrm{~d} x \text {. }
$$

To compare these results with those of this paper, we refer to the KdV-type of eq. (69) which describes the surface elevation $\eta$ in terms of the physical variables $x$ and $t$ (for the case $\alpha=1$ as considered by Newell). If we rewrite this equation in the variables $\tau$ and $\Theta$ used by Newell, there results:

$$
\eta_{\tau}+9 h^{-2} \eta \eta_{\theta}+\eta_{\theta \theta \theta}=-\frac{1}{9} \Pi \eta+O(\epsilon),
$$

which completely agrees with (91) in the required order of accuracy.

Moreover, the energy and mass functional $(77),(78)$ that are conserved for (69), transform to the conserved functionals (92) (the mass functional is the same; the energy functional has a different form because of the two different sets of independent variables, $(x, t)$ and $(\Theta, \tau)$ respectively, that are used).

Concluding we can say that our result, for the wave height $\eta$ in the physical variables $x$ and $t$, agrees with the result of Newell when the latter one is applicable, i.e. for $\alpha=1$.

\section{Conclusions}

We derived a new version of a forced $\mathrm{KdV}$ equation for unidirectional surface waves over uneven bottom. When the bottom variations are mild enough, the resulting equation (69) has a Hamiltonian structure as a consequence of the fact that the structure map $\Gamma(x)$ is skew-symmetric. The main effect of this property is demonstrated to the lowest order equation (55) in Section 4.2. For this linear equation the quadratic energy is conserved. This is in contrast to an equation like

$$
\partial_{t} \eta=-c(x) \partial_{x} \eta
$$

which is valid to the same order i.e. o(1), since $\Gamma(x)=c(x) \partial_{x}+\mathrm{O}\left(\epsilon^{\alpha}\right)$. However, the quadratic energy is not conserved for this equation:

$$
\partial_{t} \int \frac{1}{2} \eta^{2}=\frac{1}{2} \int c^{\prime}(x) \eta^{2}(x) .
$$

The energy is decreasing during run up. This can also be seen from the exact solution of (94) which is given by

$$
r(x, t)=g(\sigma(x)-t), \text { with } \sigma^{\prime}(x)=\frac{1}{c(x)} .
$$

Compared to (56) the difference is in the factor $1 / \sqrt{c(x)}$, which causes an increase in amplitude.

In the literature, equations are derived for uni-directional waves over localized disturbances of a flat bottom, see references quoted above. They are not necessarily expressed in the surface elevation itself and they are of the form of the standard KdV equation with some forcing added. In the modified 
$\mathrm{KdV}$ equation derived here, the forcing can be understood from Fig. 6. Note that in particular, for localized unevenness, after passage of the lowest order wave, there remains a forcing (localized at the unevenness) when $\mathcal{R} \neq 0$. However, when bottom variations are smooth (different from the illustration in Fig. 6) the integral of the forcing always vanishes:

$$
\int_{a}^{b} \mathcal{F} \mathrm{d} x=-\frac{1}{4} \int_{a}^{b} \partial_{x}\left(\sqrt{c(x)} c(x)^{\prime}\right) \mathcal{R} \mathrm{d} x=0 .
$$

For a localized initial wave, the remainder $\mathcal{R}$ is given explicitly by

$$
\mathcal{R}=-\int_{x_{-}}^{x_{+}} \frac{\eta_{0}(x)}{\sqrt{c(x)}} \mathrm{d} x
$$

If the initial wave is above a flat part of the bottom, $\mathcal{R}$ vanishes or not, depending on $\int \eta_{0}$ being zero or not. So, for disturbances of a still water level which conserve "mass": $\int \eta_{0}(x) \mathrm{d} x=0$, there will be no forcing and no tail wave (see Section 5.3) left after passage of the unevenness, which is in agreement with natural expectation.

\section{Acknowledgement}

A preliminary version of parts of this paper has been prepared together with $H$. Tandra, and published in the proceedings of a symposium at ITB in 1991 [15]. The authors would like to thank $\mathbf{H}$. Tandra for useful discussions. One of us (EvG) thanks the Mathematics Department of ITB and the IUC- Engineering of Bandung for the hospitality and funding of the visit which initiated this research.

\section{References}

[1] V.E. Zakharov, "Stability of periodic waves of finite amplitude on the surface of a deep fluid", J. Appl. Mech. Tech. Phys. 2, 190-194 (1968).

[2] L.J.F. Broer, "On the Hamiltonian theory of surface waves", Appl. Sci. Res. 29, 430-446 (1974).

[3] L.J.F. Broer, E. van Groesen and J. Timmers, "Stable model equations for long water waves", Appl. Sci. Res. 32, 619-634 (1976).

[4] P.J. Olver, "Unidirectionalization of Hamiltonian waves", Phys. Lett. A126, 501-506 (1988).

[5] F. Mattioli, "Decomposition of the Boussinesq equations for shallow-water waves into a set of coupled Korteweg-de Vries equations", Phys. Fluids A (Fluid Dyn.) 10, 2355-2359 (1991).

[6] P.J. Olver, "Hamiltonian and non-Hamiltonian models for water waves", Springer Lect. Notes Phys. 195, 273-290 (1984).

[7] C.C. Mei, The Applied Dynamics of Ocean Surface Waves, John Wiley and Sons, New York, Chichester, Brisbane, Toronto, Singapore (1983).

[8] A.C. Newell, Soliton in Mathematics and Physics, Society for Industrial and Applied Mathematics, Philadelphia, Pennsylvania (1985).

[9] R.H.J. Grimshaw and N. Smyth, "Resonant flow of a stratified fluid over topography", J. Fluid Mech. 169, 429-464 (1986).

[10] O.S. Madsen and C.C. Mei, “The transformation of a solitary wave over an uneven bottom", J. Fluid Mech. 39, 781-791 (1969). 
[11] T. Kakutani, "Effect of an uneven bottom on gravity waves", J. Phys. Soc. Jpn. 30, 272-276 (1971).

[12] R.S. Johnson, "On the development of a solitary wave moving over an uneven bottom", Proc. Cambridge. Philos. Soc. 73, 183-203 (1973).

[13] J.W. Miles, "On the Korteweg-de Vries equation for a gradually varying channel", J. Fluid Mech. 91, 181-190 (1979).

[14] V.D. Djordjević and L.G. Redekopp, "On the development of packets of surface grafity waves moving over an uneven bottom", J. Appl. Math. Phys. 29, 950-962 (1978).

[15] S. Redjeki, H.Tandra and E. van Groesen, "Korteweg-de Vries for surface waves over an uneven bottom", Proc. Institut Teknologi Bandung 24(1), Supplement, 179-191 (1991). 